\title{
Estudio socioeconómico del grupo de discapacitados de la Parroquia Macas para promover la inserción laboral
}

\section{Socio-economic study of the disabled group of Macas Parish to promote labor insertion}

\author{
Delgado Gomezcoello, Carlos Javier ${ }^{1}$ y Durán Parra, Dayana Rubi ${ }^{1}$ \\ ${ }^{1}$ Universidad Católica de Cuenca, Sede Macas, Ecuador \\ *cdelgadog@ucacue.edu
}

\begin{abstract}
Resumen
La inserción laboral de la población y formar parte tanto social como económicamente de la sociedad, es considerado un punto clave para la integración de las personas que tienen una incapacidad. El planteamiento de este artículo fue detallar y examinar correspondientemente los problemas y las principales estrategias para la inserción laboral de este grupo social y de esta forma hacer cumplir con sus derechos establecidos por parte del Estado ecuatoriano. Se estudió a las personas del cantón Morona parroquia Macas y a las instituciones del sector, aplicando 45 encuestas al grupo social, 5 encuestas a los diferentes establecimientos de la zona y 2 entrevistas a las principales instituciones involucradas con la inserción laboral y discapacidades en nuestro país. La investigación nos refleja con respecto a las instituciones que las mismas si cumplen con en el artículo 42 numeral 33 del Código del Trabajo ya que estas deben incluir laboralmente a personas con discapacidad según sea su número de trabajadores, se analizó de igual forma el modelo de Empleo con Apoyo que se da como una estrategia aprobada a nivel internacional la cual cuenta con una alta evidencia empírica apoyada en publicaciones y en resultados investigativos para la inserción laboral de este grupo social, que por dichos resultados pudiera ser adaptada a nuestro país y de esta forma contribuir con este grupo prioritario. Con el apoyo laboral para la mejora de estas personas se cambiará su calidad de vida y la de su hogar, exponiendo su talento, creatividad, dignidad, personalidad, autoestima, aptitudes mentales y físicas, y su desarrollo colaborativo dentro de una empresa, aportando positivamente a la sociedad de la parroquia.
\end{abstract}

Palabras clave: Inserción laboral, discapacidad, grupo social, empleo con apoyo..

\begin{abstract}
The labor insertion of the population and being part both socially and economically of society, is considered a key point for the integration of people who have a disability. The approach of this article was to detail and to examine correspondingly the problems and the main strategies for the labor insertion of this social group and in this way to enforce with their established rights on the part of the Ecuadorian State. We studied the people of the Morona canton parish Macas and the institutions of the sector, applying 45 surveys to the social group, 5 surveys to the different establishments in the area and 2 interviews to the main institutions involved with labor insertion and disabilities in our country. The investigation reflects us with respect to the institutions that the same if they comply with article 42 numeral 33 of the Labor Code since these must include labor disabled people according to their number of workers, the model of analysis was similarly analyzed Employment with Support that is given as a strategy approved at international level which has a high empirical evidence supported by publications and research results for the labor insertion of this social group, which for these results could be adapted to our country and from this contribute to this priority group. With the work support to improve these people will change their quality of life and that of their home, exposing their talent, creativity, dignity, personality, self-esteem, mental and physical skills, and their collaborative development within a company, contributing positively to the society of the parish.
\end{abstract}

Key words: Labor insertion, disability, social group, employment with support..

\section{Introducción}

En la actualidad el trabajo es el primordial motor para la inclusión de las personas con discapacidad en la sociedad, promoviendo de esta forma la inserción laboral de las mismas.
En este sentido al no disponer de un empleo, las personas además de perder su fuente de ingresos, ven amenazadas la realización de proyectos que garanticen su plena autonomía. Los instrumentos de integración de las personas con discapacidad obedecen a dos modalidades: integración laboral 
ordinaria y especial. La primera hace referencia a todas las medidas que facilitan la contratación de estas personas en puestos de trabajo habituales, es decir, no diseñados específicamente en función de la discapacidad del trabajador, con lo que se persigue remover obstáculos para lograr la igualdad en el acceso al empleo; con el fomento de estas iniciativas, los poderes públicos aplican medidas de discriminación positiva, tales como las cuotas de reserva de empleo, contratos específicos de fomento del empleo, incentivos económicos para las empresas que contratan personas en situación de discapacidad, reducciones en las aportaciones a la Seguridad Social, etc. Por su parte, la integración laboral especial comprende todas las medidas dirigidas a la consolidación de puestos de trabajo en contextos laborales adaptados específicamente a las características de las personas con discapacidad, medidas que tienden a favorecer especialmente a los casos más graves o particulares; entre los mecanismos existentes, se puede señalar los centros especiales de empleo, los contratos específicos, los incentivos económicos y las deducciones fiscales a estos centros, etc. (Ministerio de desarrollo social, 2014, p.2).

El presente estudio sobre la situación socioeconómica del grupo de discapacitados de la parroquia Macas del Cantón Morona, la discapacidad es una temática que afecta a 431.609 ecuatorianos (SISEcuador, RIPS, 2014), que se encuentran distribuidas 108.270 en el sector rural y 323.339 en el área urbana (SISEcuador, RIPS, 2014), dentro del cantón Morona existen 98 personas discapacitadas de las cuales 82 pertenecen a la parroquia Macas (SISEcuador, RIPS, 2014), de las cuales 45 se encuentran en la posibilidad de laborar, dentro de este problema están involucradas las familias, instituciones públicas y privadas, el estado y la sociedad mismas como responsables de ayudar a que estas personas tengan una mejor calidad de vida. Para ello es necesario diagnosticar la situación actual y de las características de los miembros del grupo social de discapacitados, otro dato importante el establecer el nivel de empleabilidad de las personas con discapacidad tanto en las instituciones públicas como privadas de acuerdo al Artículo 42 numeral 33 del Código del Trabajo, con la finalidad de proponer un modelo que ayude a las personas con discapacidad a lograr la inserción laboral.

El tema tiene real importancia ya que las autoridades a nivel de Estado han definido una serie de programas de ayuda social para estas personas a más de contar con instituciones que se dedican a velar por el cumplimiento de las políticas gubernamentales en cuanto a la discapacidad.

De aquí la importancia que adquieren para la familia, la sociedad y la economía cualquier programa que tienda a reinsertar laboralmente a personas de este grupo. Es indiscutible que, si se logra volverlos productivos, no sólo dejan de gravitar negativamente en la familia y en la sociedad, sino que estarán contribuyendo a mejorar el nivel de vida de todos a través de los bienes y productos que se logren con su trabajo.

\section{Metodología}

La metodología que se llevó a efecto en el presente estudio, tuvo un enfoque cuanti-cualitativo, ya que su objetivo fue promover la inserción como efecto de la participación de las personas con discapacidad en el ámbito laboral, para lo cual se utilizaron técnicas para recabar información de la cantidad de personas que pueden ser insertadas laboralmente. El tipo de investigación descriptiva - explicativa se utilizó para detectar las principales fuentes de inconvenientes que tiene los discapacitados para lograr este objetivo, y para describir las propiedades más relevantes que explican las necesidades que tienen según los derechos de estas personas, teniendo así conocimiento suficiente para explicar y tomar decisiones correctivas, logrando un progreso laboral con los individuos que participaron en el estudio.

\section{Técnicas de investigación}

La particularidad del presente proyecto es que se realizará tanto investigación de campo como bibliográfica, en la investigación de campo se utilizó:

Encuestas. - Las cuales fueron aplicadas al grupo social de discapacitados de la parroquia Macas del cantón Morona, con la finalidad de obtener datos sobre su situación económica y social; y a funcionarios de las empresas públicas y privadas, para analizar si cumplen con las ordenanzas de la Constitución de la República del Ecuador con respecto a la inserción laboral de discapacitados.

Entrevista. - Estas fueron aplicadas a las principales instituciones involucradas con la inserción laboral y discapacidades en nuestro país.

Revisión bibliográfica y análisis documental. - Técnica para recolección de datos necesarios para el estudio, esto se realizó con fuentes de información que pueden ser secundarias tomadas de estudios e investigaciones encontradas para elaborar una recopilación de estas. Para lo cual se realizó una revisión documental en diferentes bases de datos como: Google académico, Scielo, Fuente Académica Premier, ProQuest, Academic One file, Ebsco, Redalyc.org, Etc.

\section{Resultados}

Los resultados se dan en base al análisis y el logro que presenta el desarrollo de las encuestas aplicadas siendo estas un procedimiento de la investigación para recopilar datos a través de un cuestionario que fue diseñado para el grupo social de discapacitados de la parroquia Macas conformado por 45 personas. Se inician la muestra de estos resultados con el análisis de los aspectos socioeconómicos de este grupo social.

Las encuestas aplicadas en su mayoría fueron contestadas por las personas con discapacidad representando el $60 \%$ (27 personas), mientras que el $40 \%$ (18 personas) 
restante fueron contestadas por familiares o personas conocidas de la persona con discapacidad. Del total de personas del sexo masculino están representadas por el $53 \%$ de la población mientras que el $47 \%$ son del sexo femenino, los datos obtenidos con respecto a las edades nos demuestran del total que 15 se encuentran en un rango de edad de 20 a 30 años, 11 se encuentran en edades entre 31 y 40 años, 14 personas tienen de 41a 50 años y 5 personas están en el rango de edad de más de 50 años concluyendo que existen una mayoría de personas en un rango de 20 a 30 años y de 31 a 40 años, las cuales se encuentran en una edad apropiada para iniciar actividades laborales en su beneficio o de su familia.

El resultado obtenido en el tema de discapacidad nos demuestra que, del total de personas encuestadas, $45 \%$ de ellas tiene una discapacidad Física, el $31 \%$ posee una discapacidad Intelectual, el $9 \%$ discapacidad Visual, $9 \%$ discapacidad Auditiva y con un $1 \%$ discapacidad Psicológica, discapacidad Psicosocial y de Lenguaje respectivamente.

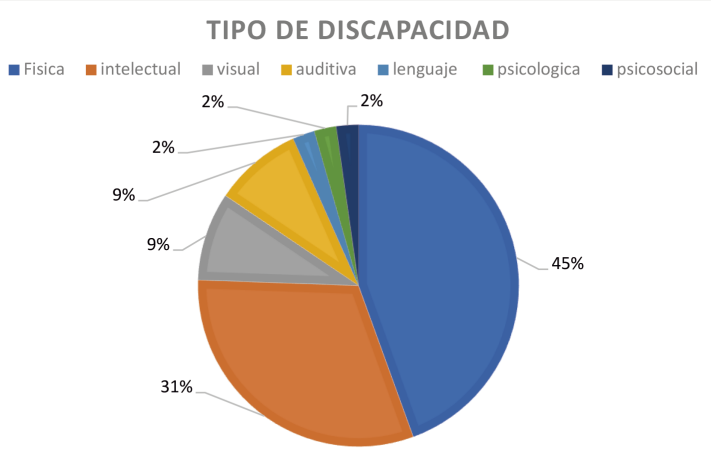

Figura 1. Tipo de discapacidad

La información obtenida acerca de la actividad que realiza la persona con discapacidad nos refleja que el $71 \%$ de los encuestados no realiza ninguna actividad mientras que el $20 \%$ trabaja de alguna manera para poder solventar los gastos que generan personalmente o familiares, el $5 \%$ dedica su tiempo al estudio y el $2 \%$ estudia y trabaja.

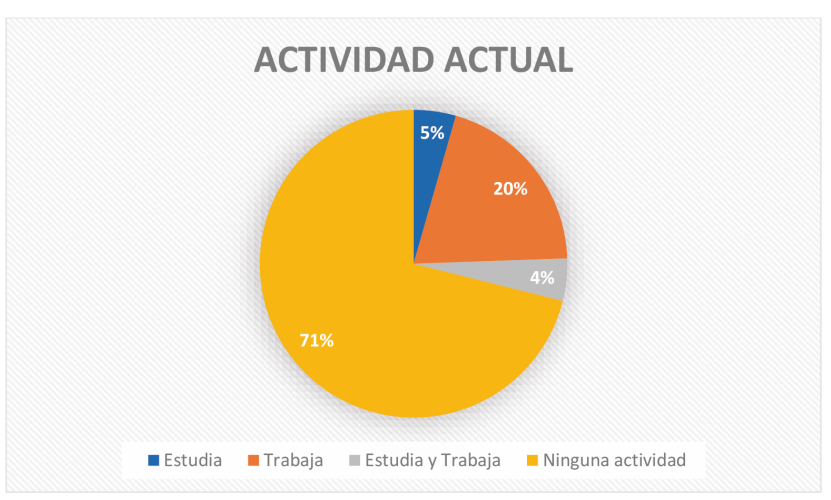

Figura 2. Actividad actual
De las personas encuestadas existe mayor cantidad de población que considera que las personas con discapacidad gozan de una igualdad de oportunidades con respecto al derecho a la educación representado por el $69 \%$, lo contrario opinan el $27 \%$ de los encuestados considerando que la educación no es de igual manera para todos ya que existen varias personas que no tienen acceso a recibir una educación individualizada acorde a su discapacidad y de esta forma no se cumple con uno de sus derechos y el $4 \%$ manifiesta que desconoce si se da una igualdad de oportunidades.

\section{EDUCACIÓN}

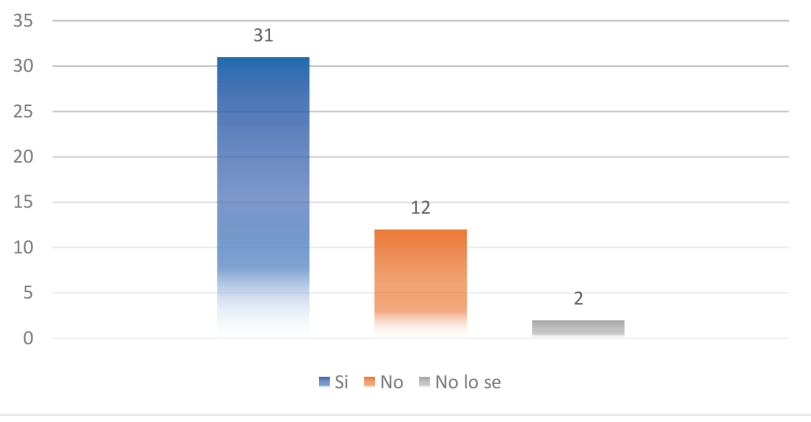

Figura 3. Educación

La información de la tabla nos demuestra que 25 personas del total encuestadas, desconoce si las personas con discapacidad ejercen sus derechos laborales con igualdad de condiciones que las demás, 13 de las encuestadas consideran que no se da esta igualdad de derechos y el 7 de las mismas afirma que se ejercen dichos derechos sin discriminación alguna con un ambiente laboral sano para sus condiciones.

Tabla 1

Igualdad de derechos

\begin{tabular}{|l|r|}
\hline $\begin{array}{c}\text { Derechos laborales } \\
\text { en igualdad de condiciones }\end{array}$ & \# de personas \\
\hline $\mathrm{Si}$ & 7 \\
\hline $\mathrm{No}$ & 13 \\
\hline No lo se & 25 \\
\hline Total & 45 \\
\hline
\end{tabular}

Analizar si las personas con discapacidad ejercen su derecho a tener un nivel de vida adecuado en igualdad de condiciones nos permite distinguir si su nivel o calidad de vida es bueno en un ambiente apropiado. Los resultados demuestran que, de las personas encuestadas, el $71 \%$ de ellas considera que las personas con discapacidad ejercen su derecho a tener un nivel de vida en igualdad de condiciones que gracias al gobierno este grupo social prioritario ha mejorado su calidad de vida gracias a las ayudas económicas y materiales que se brindan pueden vivir mejor, pero no 
en su totalidad es decir la ayuda no es totalmente suficiente, y mientras que el $29 \%$ de la población encuestada opina que sus derechos a una mejor calidad de vida no han sido bien atendidos ya que sus necesidades son grandes y no están cubiertas en su totalidad.

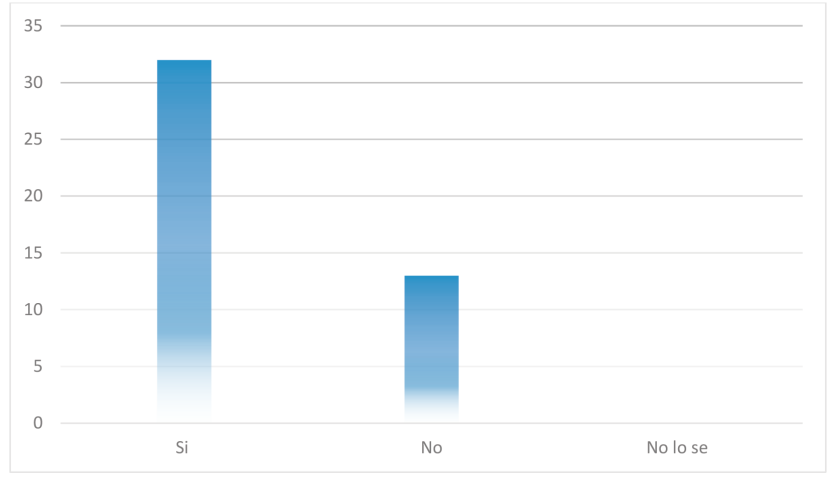

Figura 4. Derecho a un adecuado nivel de vida

Al identificar los principales problemas que se dan para la inserción laboral de este grupo social se sabe donde se debe actuar para poder eliminar estas barreras que perjudican para cumplir sus derechos.

Los datos de la encuesta nos indican que la población en su mayoría considera que los perjuicios y barreras sociales son el principal problema que se presenta representado por el $33 \%$ de los encuestados, seguido de las carencias de formación y falta de experiencia que está representado por el $29 \%$ de la población, el siguiente punto considerado es la accesibilidad al puesto de trabajo el cual está representado por el $25 \%$ de las encuestas, los puntos menos relevantes son la falta de información y sensibilización sobre la discapacidad con el $11 \%$ y por último el proteccionismo familiar con el $2 \%$ de las encuestas.

Tabla 2

Problemas de inserción laboral

\begin{tabular}{|l|r|}
\hline \multicolumn{1}{|c|}{$\begin{array}{c}\text { Problemas para la } \\
\text { inserción laboral }\end{array}$} & \multicolumn{1}{c|}{$\begin{array}{c}\text { de personas } \\
\text { La falta de accesibilidad al } \\
\text { puesto de trabajo. }\end{array}$} \\
\hline El proteccionismo familiar. & 11 \\
\hline Los perjuicios y las barreras sociales. & 15 \\
\hline $\begin{array}{l}\text { Las carencias de formación } \\
\text { y falta de experiencia. }\end{array}$ & 13 \\
\hline $\begin{array}{l}\text { La falta de información y sensibilización } \\
\text { sobre la discapacidad. }\end{array}$ & 5 \\
\hline TOTAL & 45 \\
\hline
\end{tabular}

Analizar la opinión reflejada en el total de las encuestas nos indica que las personas con discapacidad consideran la principal opción para mejorar la inserción laboral es la creación de talleres de orientación laboral y búsqueda activa de empleo específico para personas con discapacidad opción apoyada por el $34 \%$ de la población encuestada, seguida se encuentra el facilitar el acceso a la formación respaldada por el $23 \%$ de la población, opciones que se deben tomar en cuenta para poder lograr su inserción ya que no han sido atendidas en un $100 \%$ para cumplir con su derecho.

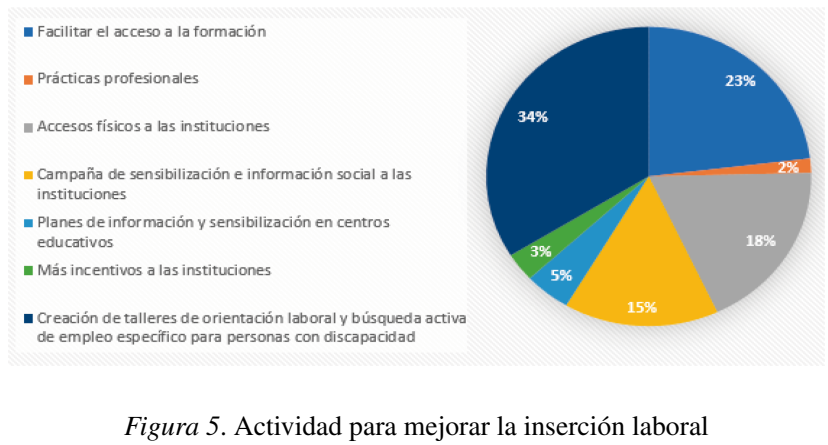

Las respuestas señalan que el $49 \%$ de los encuestados consideran que uno de los motivos por los cuales no consiguen trabajo es porque las empresas no les dan las oportunidades necesarias, ya que la adecuación para su área de trabajo es un costo que las instituciones no quieren adquirir, dificultando así la inserción laboral de este grupo social, en el mismo porcentaje se encuentra que el motivo es porque la sociedad pone barreras, se ve presente la discriminación hacia los mismos menospreciando sus capacidades sin brindarles oportunidad de desarrollarse y superarse de manera profesional.

Tabla 3

Motivo por el cual no consiguen trabajo

\begin{tabular}{|l|r|}
\hline \multicolumn{1}{|c|}{ Alternativas } & \multicolumn{1}{|c|}{$\begin{array}{c}\text { Número } \\
\text { de personas }\end{array}$} \\
\hline $\begin{array}{l}\text { Porque las empresas no } \\
\text { les dan la oportunidad }\end{array}$ & 22 \\
\hline Porque la sociedad pone barreras & 22 \\
\hline Porque ellos no desean trabajar & 1 \\
\hline Total & 45 \\
\hline
\end{tabular}

\subsection{Datos obtenidos a partir de la entrevista}

Una de las principales instituciones que brindan ayuda a las personas con discapacidad para lograr su inserción laboral es el CONADIS (Consejo Nacional para la igualdad de Discapacidades) el objetivo de esta institución es promover la realización de políticas públicas sobre la discapacidad con la finalidad de lograr la integración económica, social y cultural de este grupo vulnerable, mediante el planteamiento de programas y proyectos a nivel de la provincia que conduzcan al cumplimiento de sus derechos.

El apoyo que la institución brinda en el tema laboral es la inserción en empresas públicas y privadas, brindan orientación laboral (charlas y talleres); cuenta con convenios con el ministerio de trabajo 
velando con el cumplimiento de la cuota laboral que deben tener las empresas. Para la selección de personas se revisa un sistema informativo donde está toda la información necesaria de cada persona con discapacidad examinando si la persona es apta para laborar. El principal problema que la institución percibe con respecto a la inserción es el tema educativo ya que las empresas solicitan personas que tengan una educación mínima de bachilleres, por lo que se concluye que se debe prestar más atención al tema de educación en este grupo vulnerable que ayudara para lograr su inclusión, seguido del problema educativo se presenta la edad de las personas con discapacidad, otro obstáculo que ponen las empresas ya que en su mayoría estas personas son contratadas para labores de limpieza por lo que se tiene preferencia por personal joven que pueda cumplir estas tareas sin mayores inconvenientes. (Cordero, 2017)

\subsection{Modelo para lograr la inserción laboral de las perso- nas con discapacidad}

\subsubsection{El Empleo con Apoyo}

El empleo con apoyo, es una modalidad con el objetivo de conseguir la inserción de las personas con discapacidad en el mercado de trabajo, el empleo con apoyo consiste en formar a una persona para un puesto determinado. (García A., Empleo para las personas discapacitadas, 2007)

Para esta actividad se cuenta con un preparador laboral, que le enseña a desempeñar su labor, le acompaña al puesto de trabajo y, una vez concluido este proceso, realiza un seguimiento a largo plazo de su situación en la empresa. En definitiva, se trata de conseguir que las personas discapacitadas accedan a un empleo remunerado en el mercado libre, en las mismas condiciones que el resto de trabajadores y trabajadoras. (García A., Empleo para las personas discapacitadas, 2007)

\subsubsection{Beneficios del Empleo con Apoyo}

Los beneficios de esta modalidad son tanto para la persona beneficiaria, como para las empresas y la sociedad, a la que permite su participación en el desarrollo social. (García A., Consumer, 2007)

Esta modalidad incluye una amplia gama de puestos de trabajo, desde auxiliares administrativos a reponedores en un supermercado o limpiadores. En todos los casos, el proceso de aprendizaje es personalizado y adaptado a cada trabajador o trabajadora, por lo que se le instruye en unas labores determinadas, si bien se intenta no caer en la rutina. (García A., Empleo para las personas discapacitadas, 2007)

Elementos y a su vez tareas que configuran el perfil del profesional de la integración laboral:
El análisis del trabajo, permite mediante la recopilación de información y una observación continuada y estructurada, determinar cuáles son los elementos fundamentales del empleo que se está analizando, los procedimientos de la empresa, sus políticas de personal, los servicios disponibles en el lugar de trabajo y su configuración física. Esta tarea debe ser desarrollada con la colaboración de algún trabajador de la empresa que sin duda conocerá y dominará mucho mejor que el profesional que evalúa todo lo anteriormente mencionado. La responsabilidad del profesional será la de saber solicitar la información necesaria e indicar al trabajador que va a obtenerla la manera de recopilarla. (Borja)

El diseño y análisis de tareas, que permite descomponer aquellas tareas en las que el trabajador encuentre especial dificultad, en pequeños elementos, que faciliten la estructuración de un entrenamiento específico para la realización de las mismas. Hay que ser sin embargo especialmente cuidadosos en este aspecto para no remarcar las diferencias sobre el resto de trabajadores y compañeros, ya que el objetivo es que el empleo sea lo más normalizado posible en todos los sentidos. (Borja)

El entrenamiento de precisión y de autonomía, utilizando técnicas conductuales que faciliten al trabajador la distinción de señales naturales que le indique el avance o el cambio en una determinada tarea, y que le proporcionen el mayor grado de autonomía posible. En este sentido, se debe de proporcionar solo la asistencia necesaria para que el trabajador se centre en la tarea y aprenda la ejecución adecuada, teniendo en cuenta sus habilidades y la variación de asistencia necesaria a lo largo del tiempo. (Borja)

El preparador laboral para el empleo con apoyo, es la figura clave del empleo con apoyo. El tiempo que deben dedicar a cada trabajador o trabajadora para ofrecerle un apoyo flexible, pero individualizado. El preparador laboral presta su ayuda al trabajador durante un periodo cercano a seis meses. La finalidad es que las personas desarrollen al máximo sus capacidades personales, sociales y laborales. La idea es que la persona que comienza un programa de empleo con apoyo en una empresa, mantenga su puesto de trabajo en el futuro. (García A., Consumer, 2007)

Muchos preparadores deben ser trabajadores sociales o diplomados en educación especial, que reciben la formación en la propia asociación para luego enseñar a los demás. La presencia del preparador, que actúa como una especie de mediador entre el trabajador y la empresa, ayuda a ésta a vencer el miedo inicial que pueda tener. (García A., Consumer, 2007)

\subsubsection{Dificultades del modelo empleo con apoyo}

Una de las principales sería la dificultad para encontrar personal cualificado y el miedo a que no cumpla el perfil requerido o no sea productivo.

Asimismo, la adaptación al puesto puede ser demasiado costosa o que no se cuente con la suficiente información sobre esta modalidad de empleo. (García A., Consumer, 2007) 


\section{Discusión y Conclusiones}

Al realizar este artículo se ha revisado diversa información acerca del grupo social de discapacitados que ayuda para conocer su estado socioeconómico y el análisis para su inserción laboral.

La investigación desarrollada ha presentado varios problemas en nuestro entorno con respecto a la inserción laboral del grupo social de discapacitados, uno de estos problemas es la falta de acceso a la educación o apoyo para la misma por lo que estas personas no se pueden desarrollar como profesionales, por tal motivo este grupo social no se quiere integrar a una institución donde podría tener oportunidades y tener una fuente de ingresos, otro impedimento para estas personas se desarrollen sería que la falta de información sobre los derechos que tienen en cuanto a lo laboral y los perjuicios sociales hacía los discapacitados, las capacitaciones y motivaciones sería algo que podría contribuir para que este caso no sea un problema de la zona, las personas con capacidades diferentes merecen ser tratadas con respeto e igualdad.

En la parroquia estudiada la mayoría de personas discapacitadas no tiene un ingreso, sólo cuentan con los bonos que perciben, pero no les es suficiente para solventar sus gastos personales, o en algunos casos familiares por ello lograr una inserción laboral con este grupo social sería de gran beneficio social y económico.

En el Ecuador una de las estrategias que podría alcanzar un mayor éxito en la incursión de las personas con alguna invalidez es el empleo en instituciones desde el 2009 en el artículo 42 numeral 33 del Código del Trabajo cada empresa o patrono personal natural están en la obligación de contratar por cada 25 empleados que tengan al $4 \%$ del total de trabajadores a personal con discapacidad. Respecto a las instituciones se observa que los resultados de esta estrategia han sido positivos en diversas zonas de nuestro país, ya que varias personas con discapacidad se han incorporado a diferentes áreas laborales donde se les ha brindado un ambiente de integración sin discriminación derivando perjuicios, reduciendo los procesos de estigmatización, observando más autonomía y autoeficacia.

Según el abogado Miguel Laloma García, el Empleo Protegido . ${ }^{\text {es }}$ aquel que ha sido generado para personas con discapacidad en empresas ordinarias que cumplen determinadas características orientadas a facilitar la incorporación de trabajadores con discapacidad al mercado laboral". (Tapia, Castro, \& Aguilera, 2013)

El objetivo principal de este tipo de empleo es la realización de un trabajo productivo, la participación en operaciones de mercado y la mantención de una remuneración. Estos empleos han sido concebidos para personas que presentan alguna discapacidad, un bajo nivel de funcionamiento social y que adolezcan de una preparación suficiente como para participar en espacios laborales normalizados. Respecto a las remuneraciones, éstas sólo ocasionalmente se correlacionan con la calidad de los productos confeccio- nados o con el logro de las tareas realizadas y suelen ser de baja cuantía. (Tapia, Castro, \& Aguilera, 2013)

El empleo con apoyo constituye una de las actuales alternativas al trabajo protegido. Aunque el número de servicios de empleo con apoyo para personas con discapacidad ha aumentado de forma significativa en los últimos años, su extensión no ha ido acompañada de estudios que permitan esclarecer que dimensiones, qué factores favorecen los procesos de inserción de las personas con discapacidad en el mercado laboral ordinario. (Pallisera, Es, Fullana, Es, \& Es, 2005)

La investigación de (Serra, 2004) pretende “conocer en profundidad el proceso de adaptación laboral de los trabajadores con discapacidad a partir de los tres principales protagonistas: el trabajador, el preparador laboral y el compañero/supervisor laboral" que sea beneficioso para su familia.

El objetivo laboral es determinar la incidencia y el funcionamiento de los principales agentes de apoyo en el proceso de inclusión laboral en la empresa normalizada.

Según (Alomar, 2004) es probable que se llega a un mejor resultado el: Análisis de un estudio de casos, el comportamiento de las variables personales, laborales y sociales que puedan ayudar a predecir el éxito en el ajuste de un trabajador con discapacidad en una empresa ordinaria con la modalidad de Empleo con apoyo. (p.1)

Finalmente, concluimos que, en el proceso de inserción laboral de las personas con discapacidad, se necesita tomar medidas que acrediten el acceso y la permanencia de la persona en un determinado lugar de trabajo, respetando su individualidad y el tipo de discapacidad. Para alcanzar una inserción laboral se debe empezar mejorando calidad y el acceso a la educación y la formación ocupacional.

Los efectos del trabajo de investigación nos permiten deducir que al referirnos a las personas con capacidades diferentes hablamos de un tema importante como lo es los derechos humanos, en el Ecuador se trata como un grupo prioritario para el cual se ha implementado políticas, y en la orientación de la inserción laboral se ha mostrado mayor eficacia en la obtención de empleo competitivo es el empleo en instituciones para personas con discapacidad en nuestro país, o que se pueda dar la posibilidad que se dé el empleo con apoyo como en otros países, ya que en comparación con lo propuesto por el gobierno de empleos en instituciones, el Empleo con Apoyo permite mayores fuentes de empleos, mejores remuneraciones, más horas semanales trabajadas y mayor tiempo de permanencia en el trabajo obtenido. Todo esto ayudará a las personas con capacidades diferentes a en su mejora en el nivel de funcionalidad social, a romper con los estigmas hacia este grupo social, mejorará su calidad de vida y la autoestima de los mismos.

\section{Referencias Bibliográficas}

A, P. (2008). El modelo social de discapacidad: orígenes, caracterización y plasmación en la convención 
internacional sobre los derechos de las personas con discapacidad.

Alomar, E. (2004). El traball dels joves amb retard mental en entorns normalitzats: análisid' una realitat de treball amb suport. [Tesis doctoral].

Arraz, C. (2012). Empleo y discapacidad.

Barton, L. (1998). Discapacidad y sociedad. Ediciones Morata.

Borja, J. d. U. (s.f.). Campus.usal.es.

Casado, D. (2002). Las estrategias para el cambio proinclusión de las personas con discapacidad.

Cazar, D. R. (s.f.). Icevi.

Cordero, O. (2017). Inserción laboral.

de Lomas de Zamora, U. N. (2010). Discriminación a personas con discapacidad.

Estudio de inclusion laboral de personas en situacion de disapacidd en el estado. (2014).

García, A. (2007). Consumer.

García, F. (2002). El análisis de la realidad social; métodos y técnicas de investigación (Vol. 3ra edición.). Alianza Editorial.

Hinojosa, J. (2010). conadisecuador.

Moreno, L. (2010), vicepresidencia.

Naciones unidas(UN). (2006).

Narváez, E. (2013). Estado ecuatoriano y discapacidades.

Normas internacionales del trabajo. (2010).

Porras, R. (2008). Personas con discapacidad y acceso a servicios educativos en latinoamérica.

Puig, R. (2013). La tecnología de la información en la educación especial.

Serra, F. (2004). La presència del suport natural en els processos d' inclusió laboral mitjamcant el model de treball amb suport (supported employment) [Tesis doctoral].

SISEcuador. (2014). RIPS.

Tapia, F. M., Castro, W. L., y Aguilera, J. M. M. (2013). scielo.

Tapia, Y. (2012). La inserción laboral de las personas con discapacidad y su incidencia socioeconómica en el ecuador. período 2009-2011 [Tesis].

Recibido: 15 de septiembre de 2017

Aceptado: 18 de octubre de 2017 
